\title{
SARS-CoV-2 constitution: The second and most contagious pandemic of the 21st century
}

\author{
Otun Saha ${ }^{1}$, Md. Mahbubur Rahaman ${ }^{2}$, Afroza Sultana $^{3}$, and Md. Mizanur Rahaman ${ }^{1,4}$ \\ ${ }^{1}$ Dhaka University \\ ${ }^{2}$ Stamford University Bangladesh \\ ${ }^{3}$ Noakhali Science and Technology University \\ ${ }^{4}$ University of Dhaka
}

June 1, 2020

\begin{abstract}
SARS-CoV-2, appeared in winter 2019, in Wuhan State of Hubei Province in China, which has already marched more than 210 countries with 5,418,496 cases and 344,206 fatalities. Through an ongoing unprecedented global public health effort, the pandemic is now slowing down slightly after 6 months of its original occurrence. Until now, the media coverage is incredibly accurate and has been providing worldwide pictures to enrich scientific data. As of May 25, 2019, there are more than 21000 citations related to SARS-CoV-2 in the medical literature according to the WHO on global research on coronavirus disease 2019 (COVID-19). However, among these citations, only 5-10\% are related to laboratory experimental data. This review is aimed to analyze the unique laboratory experiment aspects of SARS-CoV-2, to describe the epidemiology, threat to pediatric and women health, mutational intimidation, diagnostics and treatment of the SARS-CoV-2 in regard to future epidemics, and to use the SARS-CoV-2 experience as a model for future pandemics.
\end{abstract}

\section{DISCUSSION}

The first transmission of SARS-CoV-2 occurred in Wuhan City, Hubei Province in China [1,2]. In December 2019, a novel coronavirus (SARS-CoV-2) namely Wuhan-Hu-1 was isolated from patients with COVID-19 and subsequently sequenced (GenBank accession number MN908947.1) [3]. This virus was rapidly identified and characterized by a combination of classical virological methods and cutting-edge molecular biology. Real time reverse transcription polymerase chain reaction (RT-PCR) based examination followed by nextgeneration sequencing $[4,5]$, scanning electron microscopy (SEM) [5] examination of upper respiratory tract (URT) swabs (nasal, throat) and sputum specimens from affected patients revealed the presence of viral particles. Fortuitously, this newly identified agent replicated in Vero cells as like as SARS-CoV, in contrast to other human coronaviruses. Although along with that of others deposited in the viral sequence database revealed that this newly novel SARS-CoV-2 virus are closely related to the SARS- CoV [4,5], although the pathogenicity is significantly lower than SARS-CoV (10\%) and MERS-CoV (40\%) [6]. But the genetic sequence of this COVID19 virus showed more than $80 \%$ identity to SARS-CoV and $50 \%$ to the MERS-CoV $[4,5,6]$. Cytopathic effect of SARS-CoV-2 in the Vero cell cultures was confluent 3 days after inoculation [7]. In a technological Blitzkrieg, the genome was completely and rapidly sequenced by several laboratories around the world. Until now 30789 complete genome were deposited in the Global Initiative on Sharing Avian Influenza Data (GISAID) (https://www.gisaid.org/). SARS-CoV-2 have the largest genomes found in any RNA virus. The genome of SARS-CoV-2, ranging from 26 to 29 kilobases in length, containing 14 open reading frames (ORFs) [8] encoding 27 proteins [9]. Moreover, the spike which is divided into the S1 domain (responsible for receptor binding) and S2 domain (responsible for cell membrane fusion ) surface glycoprotein plays an essential role in binding to receptors on the host cell and is crucial for determining host 
tropism and transmission capacity, mediating receptor binding and membrane fusion [10]. Furthermore, the eight accessory proteins (3a, 3b, p6, 7a, 7b, 8b, 9b, and orf14) and four major structural proteins, including the spike surface glycoprotein $(\mathrm{S})$, small envelope protein $(\mathrm{E})$, matrix protein $(\mathrm{M})$, and nucleocapsid protein $(\mathrm{N})$, are located in the 3'-terminus of the SARS-CoV-2 genome [9]. These sequences data not only permitted the rapid development of highly specific diagnostic tests, but also helped in the epidemiologic tracking of the pandemic followed by development of rapid treatment process. Moreover, cataloging the genome from human cases assisted in the search for the origin of this disease, when viruses related to the SARS-CoV-2 were identified in animals Rhinolophus affinis (Bat), Rhinolophus malayanus (Bat), Manis javanica (Pangolin) in a live animal market in Wuhan, China [11]. Viral genomes from swabs from Rhinolophus affinis and Manis javanica (Pangolin) [11] were $96 \%$ and $91.02 \%$ homologous respectively to the human SARS-CoV-2, and represented a distinct phylogenetic group from the human isolates suggesting animal to human transmission $[3,11]$. The genome sequence of SARS-CoV-2 shows that Orf8 is intact. As the pandemic progressed, most human isolates contained a SARS-CoV-2 sequence with a deletion of 382 nucleotides in this open reading frame [12]. Signature sequences were also identified in the amino acid sequences of the spike protein, which is involved in the attachment of viral particles with lung epithelial cells primarily and angiotensin-converting enzyme 2 (ACE2) receptor [3, 9, 11, 12]. Khailany et al., (2020) [13] revealed that 116 mutations were present in studied 95 SARS-CoV-2 genomes where the three most common mutations were $8782 \mathrm{C}>\mathrm{T}$ in ORF1ab gene, 28144T $>\mathrm{C}$ in ORF 8 gene and $29095 \mathrm{C}>\mathrm{T}$ in the $\mathrm{N}$ gene. In another phylogenetic networks analysis by Forster et al., (2020) [14] distinguished 3 major circulating variants (A, B, C) in the basis of amino acid variation among the genome sequences of SARS-CoV-2 in which A being the ancestral type according to the bat outgroup coronavirus. G29553A was found to be almost exclusive to the all US isolates leading to amino acid substitutions in the viral protein sequence are absent in the isolates from Italy and Spain in the SARS-CoV-2 genomes deposited in GenBank and GISAID [13, 14]. Most alarmingly, one study handling on large number of deposited genomes found that among all (198) independent mutation (homoplasies), $80 \%$ recurrent mutations produced non-synonymous changes at the protein level, suggesting possible ongoing adaptation of SARS-CoV-2 [15]. However, SARS-CoV-2, like other RNA viruses, mutate rapidly as a consequence of the error-prone nature of RNA polymerases and their characteristically short replicative life cycles $[4,5,10,14]$. Thus, mutations can emerge rapidly and persist. .

Also of interest, the first case of COVID-19 has reported in a patient in Wuhan who had link to the seafood market [16] and also mainly bats are reported to be rare in markets in China [15, 16]. Although the SARSCoV-2 may theoretically transmit directly from bats or pangolin to humans, most interestingly the evidence so far suggests it more likely spreads indirectly through another animal playing the role of an intermediate host to transmit the virus to humans, supporting the hypothesis that the SARS-CoV-2 originated from animals but actual source is still unknown [16].

The epidemiology of SARS-CoV-2 is both extremely interesting and aggressive $[10,14,16]$ than the all other reported viruses. The primary case in this outbreak was a 55-y-old woman a shrimp seller in China's Wuhan city, the originating point of the coronavirus pandemic, has been identified as one of the first victims of coronavirus. She developed a cold on 10th December and she was selling shrimps at the Huanan Seafood Market [17]. Wei was among the first 27 patients to test positive for COVID-19 and one of 24 cases directly related to the market who shared a common toilet in the market [17]. On 9th January 2020, Wuhan witnessed the first confirmed death due to COVID-19 [17] followed by the Philippines on 1st February reported first death outside China province [18], and on 14th February, France reported first death outside Asia [18]. Furthermore, due to its higher contagion nature dozens of deaths were reported in Iran, South Korea, and Italy by 28th February (COVID- 19 in Korea, 5th April 2020). Around 40 countries over all the continents, except the Antarctica, had reported deaths by 13th March, 2020 [17]. Most interestingly all of the reported $1^{\text {st }}$ cases around the world have the traveling link to Wuhan, China or any other affected countries. COVID19 then spread rapidly around the world by infected persons who traveled by airplane. Surprising, there were no data available on secondary cases acquired by co-airline passengers [19].

Since the start of this pandemic till 27th May 2020, WHO reports 5,792,992 confirmed cases worldwide with a death count of 357,480 (https://www.worldometers.info/coronavirus/) and during this time the daily 
new cases are $\sim 70,000$, and daily reported new deaths are more than 4,000 However, actual case count is likely to be much higher than the reported count owing to lack of testing, in general; and especially for the asymptomatic patients [20]. Likewise, the actual death count is much higher because as per the standard official protocols, the death accounted in the report is only of people who were tested positive for COVID -19 [20]. On the other hand, as of 27th May 2020, total of 2,499,168 cases have been recovered from COVID -19 across the world as per the data collected by John Hopkin University, USA. However, t ratio of reported death versus reported case in the top 10 countries varied between $18.8 \%$ to $1.0 \%$. Such a huge variation in the mortality rate seems to be governed by various factors like population characteristics (sex, age, and overall health), time since the initial outbreak, healthcare system, and the volume of testing and treatment options $[1,2]$.

The transmissibility of SARS-CoV-2 and human disease COVID-19 is a paradox. On the one hand, in the initial phases of the spread of SARS-CoV-2 in China, a disproportionate number of health care workers became ill and apparent "super spreader" cases were noted around the world further $[4,5,21]$. In contrast, the secondary illness rate in households was only $19.3 \%$ to $13.8 \%$ in a Guangzhou study [22]. These householdcontact rate was higher than the nonmodel-based estimates $14.9 \%$ for the Shenzhen and $10.2 \%$ for Guangzhou in another two studies [22, 23]. These household-contact rates are significantly less than other respiratory infections such as pertussis and measles. On the other hand, for SARS-CoV, the secondary attack rate was estimated to be $4.6 \%$ in Beijing, $8 \%$ in Hong Kong, $6.2 \%$ in Singapore, and $10.2 \%$ in Toronto [24]. Unfortunately information on household transmissibility of MERS-CoV is less clear [24]. Most interestingly, most other instances of super spreading involved contact by infected personnel or non-living objects or hospital personnel who gave care and had contact with the primary cases without carrying out infection control precautions (Figure 1) [4, 5, 21].

The incubation period of SARS-CoV-2 is 2-14 d, with a median of $5.2 \mathrm{~d}$ [25] (Figure 2). The attack rate in children is reported to be less than that of adults, but it appears that male (71\%) have higher rates than women. A median age of 40 years have been observed so far [21] although approximately $80 \%$ of COVID-19 deaths were reported in the patients over 60 years old (Yee et al., 2020). Over $75 \%$ of COVID-19 deaths had comorbid medical conditions such as hypertension, diabetes, or cardiovascular disease [21]. The median time from onset of symptoms to first hospital admission, and eventually to ICU admission was 7.5 days $(4 \cdot 0-8 \cdot 0)$, and 11 days $(8 \cdot 0-17 \cdot 0)$ respectively [25]. Clinical disease in children is clearly less severe than disease in adults [25]. Adolescents have illness similar to adults, but the case fatality rate in adolescents is significantly less. Although there is evidence that unrecognized infections occur, there is vast reported instance of a possible asymptomatic infection with the SARS-CoV-2 [25].

Details of clinical illness in children have been presented from studies in China, Italy, USA, UK, Bangladesh, India, Singapore, and Canada [26]. A study on 171 pediatric patients in China reported that after 2-14 days incubation period of children, the following clinical findings were noted: cough (48.5\%), pharyngeal erythema $(46.2 \%)$, fever $(41.5 \%)$, fast breathing $(28.7 \%)$, diarrhea $(8.8 \%)$, rhinorrhea $(7.6 \%)$, fatigue $(7.6 \%)$, and vomiting (6.4\%) [27] (Figure 3). Frequency of asymptomatic infection, upper respiratory tract infection and pneumonia was $15.8 \%, 19.3 \%$ and $64.9 \%$, respectively in three individual studies [27]. In patients $<10 \mathrm{y}$ of age, the most common findings were fever and cough, whereas older children ([?]10 y) also had headache, myalgia, sore throat, chills, and/or berating problem [27](Cai et al., 2020). Hilar adenopathy, extensive pleural effusions, lung abscess, pneumatocele, or pneumothorax were not observed in various study on children with COVID-19 [4, 5, 21, 22, 25, 27]. Furthermore, in a study on 116 pregnant women, the most common symptoms were fever $(50.9 \%)$ and cough $(28.4 \%)$ followed by $23.3 \%$ without symptoms, $6.9 \%$ of severe pneumonia [28]. In other study with114 pregnant women,the most common symptoms were fever (87.5\%), cough $(53.8 \%)$ followed by fatigue (22.5\%), diarrhea (8.8\%), dyspnea (11.3\%), sore throat (7.5\%), and myalgia (16.3\%) (Figure 3) [29].

The most hopeful laboratory finding is blood lymphocytes count in the case of all pediatric patients. On the other hand, adults with COVID-19 usually showed a significant or progressive decrease in the absolute number of peripheral blood lymphocytes at the early stage of the disease $[4,7$, and 8$]$. This occurs in nearly 
all bad outcome adult patient cases. In one meta-analysis by Huang et al., (2020) [30] revealed that adult patients with poor outcome (84\%) and dead $(87 \%)$ have a lower lymphocyte count. The mean difference is $361.06 \mu \mathrm{L}[-439.18,-282.95]$ and this mean difference rose to $376.53 \mu \mathrm{L}$ to $377.56 \mu \mathrm{L}$ in the case of ARDS and ICU patients. Other frequently abnormal laboratory findings include thrombocytopenia (20.0\%) but most patients had a normal CBC (normal Hb, WBC and platelet count) and lactate dehydrogenase (LDH) $[21,25,29$, and 30$]$.

The initial diagnosis of SARS-CoV-2 was based on clinical and epidemiological data. Based on a few early descriptive studies of patients admitted to a Wuhan regional referral hospital with confirmed COVID-19 pneumonia, fever was the most frequent symptom among the patients $(83 \%-98 \%)$ followed by cough $(66 \%-$ $82 \%$ ) and roughly one-third of the patients had shortness of breath [21]. Less common symptoms include myalgia (11\%), rhinorrhea (10\%), headache $(8 \%)$, chest pain $(2 \%)$, and gastrointestinal symptoms $(3 \%)$ $[6,21,23]$. The vast majority of patients presented more than one symptom [4, 6, and 21]. In some other studies, the clinical presentation of COVID-19 resembles SARS-CoV characterized with fever, dry cough, and shortness of breath in most of the cases [6, 14, 21, and 25]. Cases with critical illness showed respiratory failure, septic shock, and organs failure, which require intensive care support [6, 9, 16, and 21]. Approximately $23 \%-32 \%$ of patients were admitted to the ICU, primarily for increased oxygen support [6, 16] while one-third of the patients had complications such as acute respiratory distress syndrome, acute renal injury, septic shock, ventilator-associated pneumonia $[6,16,21]$. Patients meeting any one of the three criteria: (1) dyspnea, respiratory rate $>30$ times/min, (2) oxygen saturation, $\mathrm{PaO}_{2}$ (partial pressure of arterial oxygen) $/ \mathrm{FiO}_{2}$ (percentage of inspired oxygen) ratio $<300 \mathrm{mmHg}$ will be considered severe patients, while respiratory failure, septic shock or multiple organ failure patients will be treated as critical patients $[4,6,16,21,25,30]$. Most recently some other unusual symptoms are also reported like Strokes and blood clots, COVID toes, silent hypoxia, gastrointestinal issues, delirium, skin rashes, multi organ failure, blood clots, neurologic damage (https://www.the-scientist.com/news-opinion/the-unusual-symptoms-of-covid-1967522 ).

Laboratory confirmation of a SARS-CoV-2 infection can be determined by the demonstration of serum antibody by ELISA, isolation of the virus from a clinical specimen, or the detection of SARS-CoV RNA by a reverse transcription PCR assay, CT scan [1,2], next-generation sequencing [25], scanning electron microscopy (SEM) [4] and cell culture [4, 21]. While a higher sensitivity of $97 \%$ has been reported for Chest CT compared to RTPCR $(71 \%, \mathrm{p}<.001)$, Chest CT fails to detect the asymptomatic cases. The combination of RT-PCR and Chest CT can be used routinely because of higher sensitivity $(91.9 \%, 79 / 86)$ than RT-PCR alone $(78.2 \%, 68 / 87)$ or CT alone $(66.7 \%, 54$ of 81) [31]. However, different studies on RT-PCR showed varied sensitivity depending on the specimens used, which were lung wash (93\%), sputum (72\%), nasal swabs (63\%), lung biopsy (46\%), throat swabs (32\%), feces (29\%), and blood (1\%) [10]. This variation is probably due to the increase of viral load in the respiratory specimens from week 1 to week 2 after the onset of illness, which then dropped to lower levels at week 3 and week 4 [1,21,31]. Interestingly, the viral load in stool gradually increases after $2-3$ weeks causing fecal shedding of viruses during the recovery phase [1]. Thus, fecal samples show positive results in RT-PCR for a longer period compared to respiratory specimens.

At the present time, there are two major questions that relate to COVID-19 when world is currently grappling over the management of Coronavirus Disease 2019 (COVID-19) pandemic: Will the disease reoccur? And, if it does, how should it be treated and can it be contained? So, the question of relapse or reactivation of COVID-19 must be considered carefully. Countries like China, South Korea has already reported 5 and 116 patients (https://thehill.com/changing-america/well-being/prevention-cures/492489-morecoronavirus-patients-in-south-korea-are) respectively who were considered to be cleared of COVID-19 have tested positive again, which raises the question about virus's ability to reactivate [32]. This ultimately raises the question whether this SARS-CoV-2 has got the ability of some other viruses like EpsteinBarr virus, Ebola virus and varicella-zoster virus, which are commonly known with reactivation capacity [32]. It is possible that the false-negative results in RT-PCR might mistakenly be interpreted as viral reactivation (https://tbsnews.net/coronavirus-chronicle/south-korean-scientists-conclude-people-cannot-beinfected-twice-covid-19). 
Moreover, severe disease in humans caused by animal viruses are an ever-present danger. Severe diseases acquired from animals such as rabies, Lassa fever, and Ebola hemorrhagic fever, all of which can be transmitted from person to person, have not resulted in sustained human disease. Hence, apparently SARS-CoV-2 should also be similar. But, it seems likely that aerosolization of the virus at the Wuhan sea food market, and perhaps in some nosocomial situations made the introduction of this virus different from experiences with SARS, Lassa and Ebola viruses. However, deep anxiety has emerged as there is a possibility of a future genetic recombinant virus with the SARS-CoV-2 and a human respiratory CoV such as OC43 or 229E strains or SARS-CoV or MERS. This is because of living of animals and humans in close proximity in majority of the countries around the world and infections with human $\mathrm{CoV}$ are common, dual infection seems quite possible. However, this type of recombination among different groups of $\mathrm{CoV}$ has, to our knowledge, never been documented. Far more likely, genetic recombinant between a strain of avian influenza (such as H5N1) and a circulating human strain (such as H3N2) raising a pathogenic one [21, 33]. From past experience it appears that an influenza or more deadly others new virus pandemic will occur in the next few years for which hopefully, lessons learned from the international response to SARS-CoV-2 will contribute to its control.

The pathophysiologic events in SARS-CoV-2 are not clear. It has been seen that fewer children came in contact with COVID-19 and among infected, children have mild disease. Insights in pathophysiological mechanisms of less severity in children could be important for devising therapeutics for high-risk adults and elderly. The illness in adultsalso varies depending on the person to person as well as country to country [9, 19,34]. Early closing of schools and day-care centers led to less frequent exposure and hence, lower infection rate in children. Frequency of common symptoms was lower in children compared to adults, implying more asymptomatic infections in children. Median duration of fever in children was $3 \mathrm{~d}$ compared to $10 \mathrm{~d}$ in adult patients, implying shorter illness in children. But patients with more severe disease have less steep followed by prolonged decline in viral titres having higher level of inflammatory cytokines (IL-2, IL-6, IL-7, IL-10, GCSF, IP-10, MCP-1, MIP-1A, and TNF- $\alpha$ ) than patients with mild disease [16, 30]. Moreover, critically ill people have low number of T-cells, helper T-cells, and memory helper T-cells but contain higher naïve helper T-cells compared to mild group [35]. Increase in IgG and IgM against SARS-CoV-2 antigens was seen around day 7-10 of symptoms, followed by gradual decrease in viral load [36]. That's why choice of therapeutic agents differs in different phases of illness. There are no studies of viral loads, and cytokine levels in children with COVID-19.

Drug repurposing has been applied since the beginning as there is no known therapy. Initially both antiviral (ribavirin) and anti-inflammatory treatment followed by flu related medicine, combination of broad-spectrum antibiotics, corticosteroids and convalescent plasma have been applied. There has been reports of various clinical trials including chloroquine,hydroxychloroquine, remdesivir, favipiravir, lopinavir, ritonavir, nafamostat, camostat, famotidine, ivermectin, methylprednisolone, bevacizumab etc. and supporting agents (Ascorbic acid, Azithromycin, Corticosteroids, Nitric oxide, IL-6 antagonists)(https://www.nature.com/articles/d41591-020-00019-9).. However, most of them have already failed or did not reproduce the initial successful data [37,38]. The rationale for some cases was based on the perception that severe lung damage was occurring as a result of a "cytokine storm" [37, 38]. This thought was enhanced by the fact that illness in SARS-CoV-2 was similar to illnesses in adults due to infection with avian influenza (H5N1) and SARS-CoV. Laboratory studies with H5N1 virus in tissue culture noted the induction of proinflammatory cytokines [33,38]. There is no specific antiviral treatment recommended for COVID-19, and no vaccine is currently available $[4,6,10,14,16,21]$. Different strategies can be used depending on the severity of the patient and local epidemiology. Home management is appropriate for asymptomatic or paucisymptomatic patients. The optimal duration of home isolation is uncertain, but in consideration of incubation time around 14 days without symptoms are considered sufficient to end home isolation. Some patients with suspected or documented COVID-19 have severe disease that warrants hospital care. Oxygen therapy appears to be the major treatment intervention for those patients [39]. Mechanical ventilation may be an alternative in cases of respiratory failure refractory to oxygen therapy, while hemodynamic support is essential for managing septic shock [4, 6, 16, and 39]. Besides, the empirical treatment using convalescent 
plasma collected from patients who have recovered from COVID-19 has recently yielded satisfactory results considering both mortality and morbidity [39].

Although it seems unlikely to us that pandemic infection with the SARS-CoV-2 will ever occur, there has been considerable effort to develop a vaccine $[39,40]$. As of now, eight vaccines, including one developed by the University of Oxford, are under phase II of their clinical evaluation. Besides 110 vaccines (mRNA-1273, Ad5-nCoV, AZD1222, PittCoVacc, PiCoVacc etc.) are now going through pre-clinical evaluation. The initial success has made the scientists more optimistic about effectiveness of the vaccine in humans. Once successful, the university authority expects to produce at least 1.0 million doses of vaccine by September next. To us, this seems ill advised for two reasons (https://thefinancialexpress.com.bd/views/covid-19-vaccine-hope-andreality-1589728682). First, if a reoccurrence of pandemic disease were to occur, it is likely, since its origin will be from an animal, that the new virus might be different from the present human SARS-CoV-2. Secondly, vaccines against known animal $\mathrm{CoV}$ have had varied results. Of particular concern in this regard is the possibility of enhanced SARS-CoV-2 rather than protection. This has happened before in humans with other RNA viruses (measles and respiratory syncytial virus) and it has happened in the animal setting with a feline $\mathrm{CoV}$ vaccine (Denison MR, personal communication) [33]. At the present time, the most important factor in preventing an ongoing pandemic as well as future epidemic or pandemic of SARS-CoV-2, as well as epidemics or pandemics with other more ferocious new viruses, is sound public health policy and the use of standard infection control procedures. SARS-CoV-2 gained a foothold such like today crucial scenario because of an unusual event (probable early stage not getting actual data), and the failure to recognize the problem and to initially use respiratory isolation procedures, and to use quarantine measures. A further problem is that all hospitals built still now do not have the capacity to handle large number of patients who require respiratory isolation. But unfortunately all we need to keep in mind that we have to live with COVID-19 for next few years and so we need to learn about prevention as it is the best practice in order to reduce the impact of COVID-19 considering the lack of effective treatment. In order to achieve this goal, the main measures are the following: (1) to use face masks; (2) to cover coughs and sneezes with tissues; (3) to wash hands regularly with soap or disinfection with hand sanitizer containing at least $60 \%$ alcohol; (4) to avoid contact with infected people; (5) to maintain an appropriate distance from people; and (6) to refrain from touching eyes, nose, and mouth with unwashed hands [21, 25]. Finally this review provides a clear scenario into the COVID-19 current situation and represents a picture of the current state in terms of public health impact, pathophysiology and clinical manifestations, diagnosis, case management, emergency response and preparedness. Only once this pandemic ends, one will be able to assess the health, social and economic impact of this global disaster and we should be able to learn lessons especially in terms of public and global health for any future similar pandemics.

\section{Author's contributions}

O.S., A.S. and M.M.R. carried out the studies (Data collection and data analysis). O.S., A.S and M.M.R. drafted the manuscript. M.M.R. developed the hypothesis, supervised the whole work and critically review the drafted manuscript. All authors read and approved the final manuscript.

\section{Acknowledgments}

The authors would like to acknowledge Bangabandhu Science \& Technology Fellowship Trust for supporting Otun Saha with PhD fellowship.

\section{Conflict of interest}

The authors declare that the research was conducted in the absence of any commercial or financial relationships that could be construed as a potential conflict of interest.

\section{Funding source}

No Funding 


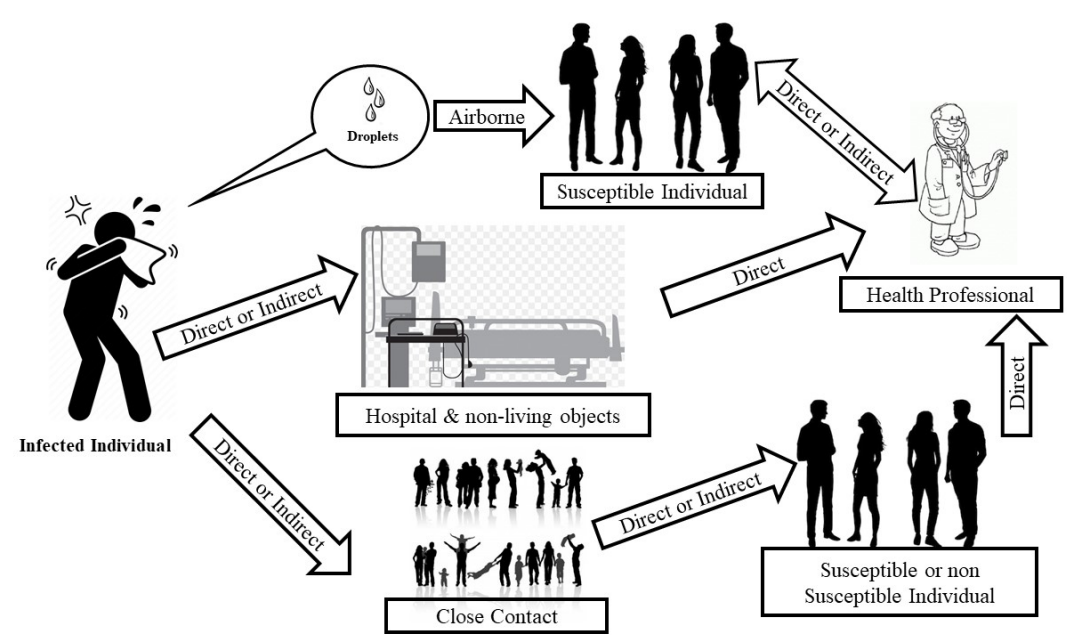

Figure 1: Possible transmission routes of pandemic 2019-nCoV in environments and hospitals

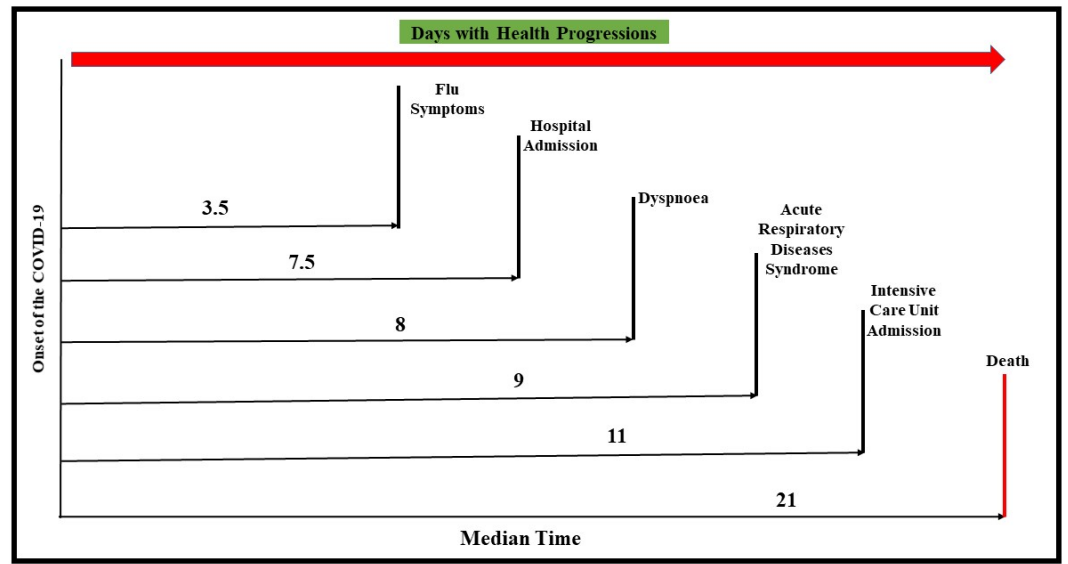

Figure 2: Timeline of 2019-nCoV cases after onset of illness. 


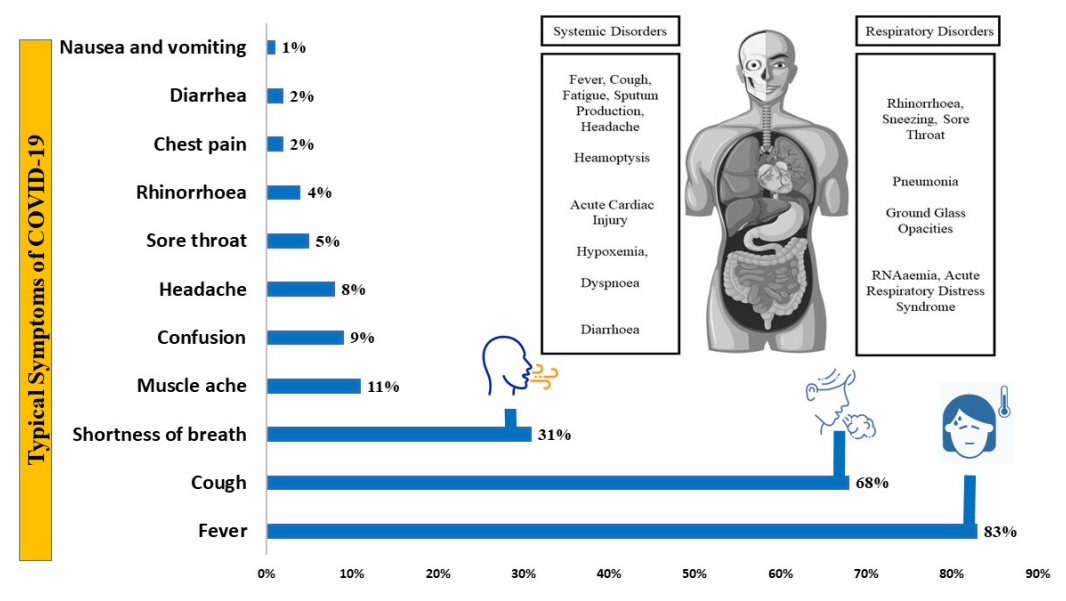

Figure 3: Representative typical Symptoms of COVID-19 with tentative percentages. A. Percent of all reported symptoms of Pandemic2019- $\mathrm{nCoV}$ affected patients between January 23 and May 25, 2020 outbreak (www.worldometers.info/coronavirus.com). b. The disorders caused by pandemic COVID-19 infection with an approximate 5.2 days incubation period. There are general similarities in the symptoms between COVID19 and previous life threading betacoronavirus SARS and MERS but COVID-19 showed some unique clinical features like rhinorrhoea, sneezing, and sore throat.

\section{References}

1. Ahamed, M. M., Naznin, R. N., Saha, O., \& Rahaman, M. M. Recommendation of fecal specimen for routine molecular detection of SARS-CoV-2 and for COVID-19 discharge criteria. Pathogens and global. Health 2020; 1.

2. Islam, M. M., Rakhi, N. N., Islam, O. K., Saha, O., \& Rahaman, M. M. Challenges to be considered to evaluate the COVID-19 preparedness and outcome in Bangladesh. International Journal of Healthcare Management 2020; 1-2.

3. Zhang, L., Yang, J. R., Zhang, Z., \& Lin, Z. Genomic variations of SARS-CoV-2 suggest multiple outbreak sources of transmission. medRxiv 2020.

4. Pang, J., Wang, M. X., Ang, I. Y. H., Tan, S. H. X., Lewis, R. F., Chen, J. I. P., .. \& Ng, X. Y. Potential Rapid Diagnostics, Vaccine and Therapeutics for 2019 Novel Coronavirus (2019-nCoV): A Systematic Review. J of Clin Med 2020; 9(3): 623.

5. Chu, D. K., Pan, Y., Cheng, S., Hui, K. P., Krishnan, P., Liu, Y., .. \& \& Peiris, M. Molecular diagnosis of a novel coronavirus (2019-nCoV) causing an outbreak of pneumonia. Clin chemistry 2020.

6. Chen, N., Zhou, M., Dong, X., Qu, J., Gong, F., Han, Y., .. \& \& Yu, T. Epidemiological and clinical characteristics of 99 cases of 2019 novel coronavirus pneumonia in Wuhan, China: a descriptive study. The Lancet 2020; 395(10223): 507-513.

7. Park, W. B., Kwon, N. J., Choi, S. J., Kang, C. K., Choe, P. G., Kim, J. Y., .. \& Seo, J. S. Virus isolation from the first patient with SARS-CoV-2 in Korea. Journal of Korean Medical Science2019; 35(7).

8. Conway, M. J. Identification of coronavirus sequences in carp cDNA from Wuhan, China. Journal of Medical Virology .2020.

9. Wu A, Peng Y, Huang B et al. Genome composition and divergence of the novel coronavirus (2019$\mathrm{nCoV}$ ) originating in China. Cell Host Microbe 2020.

10. Wang, H., Li, X., Li, T., Zhang, S., Wang, L., Wu, X., \& Liu, J. The genetic sequence, origin, and diagnosis of SARS-CoV-2. European Journal of Clinical Microbiology \& Infectious Diseases 2020; 1.

11. Zhang, T., Wu, Q., \& Zhang, Z. Probable pangolin origin of SARS-CoV-2 associated with the COVID19 outbreak. Current Biology 2020. 
12. Su, Y., Anderson, D., Young, B., Zhu, F., Linster, M., Kalimuddin, S., .. \& \& Yan, G. Discovery of a 382-nt deletion during the early evolution of SARS-CoV-2. bioRxiv 2020.

13. Khailany, R. A., Safdar, M., \& Ozaslan, M. Genomic characterization of a novel SARS-CoV-2. Gene reports , 19 , 100682. Advance online publication. 2020. https://doi.org/10.1016/j.genrep.2020.100682

14. Forster, P., Forster, L., Renfrew, C., \& Forster, M. Phylogenetic network analysis of SARS-CoV-2 genomes. Proceedings of the National Academy of Sciences 2020; 117(17), 9241-9243.

15. van Dorp, L., Acman, M., Richard, D., Shaw, L. P., Ford, C. E., Ormond, L., .. \& \& Ortiz, A. T. Emergence of genomic diversity and recurrent mutations in SARS-CoV-2. Infection, Genetics and Evolution 2020; 104351.

16. Huang, C., Wang, Y., Li, X., Ren, L., Zhao, J., Hu, Y., .. \& \& Cheng, Z. Clinical features of patients infected with 2019 novel coronavirus in Wuhan, China. The Lancet 2020; 395(10223): 497-506.

17. Kumar, M., Taki, K., Gahlot, R., Sharma, A., \& Dhangar, K. A chronicle of SARS-CoV-2: PartI-Epidemiology, diagnosis, prognosis, transmission and treatment. Science of The Total Environment 2020; 139278.

18. Ramzy, A., May. T., 2nd February 2020. Philippines Reports First Coronavirus Death Outside China. Available at https://www.nytimes.com/2020/02/02/world/asia/philippines - coronavirus -china.html

19. Yang, N., Shen, Y., Shi, C., Ma, A. H. Y., Zhang, X., Jian, X., .. \& Fu, Y. In-flight Transmission Cluster of COVID-19: A Retrospective Case Series. medRxiv 2020.

20. Reuters, 2020 b. "Special Report: Italy and South Korea virus outbreaks reveal disparity in deaths and tactics". https://www.reuters.com/article/us -health -coronaviru s -response -specialre - idUSKBN20Z27P.

21. Yee, J., Unger, L., Zadravecz, F., Cariello, P., Seibert, A., Johnson, M. A., \& Fuller, M. J. Novel coronavirus 2019 (COVID-19): Emergence and implications for emergency care. J of the American College of Emergency Physicians Open 2020.

22. Jing, Q. L., Liu, M. J., Yuan, J., Zhang, Z. B., Zhang, A. R., Dean, N. E., .. \& Lu, Y. Household Secondary Attack Rate of COVID-19 and Associated Determinants. medRxiv 2020.

23. Bi Q, Wu Y, Mei S, et al. Epidemiology and Transmission of COVID-19 in Shenzhen China: Analysis of 391 cases and 1,286 of their close contacts. medRxiv 2020. doi.org/10.1101/2020.03.03.20028423.

24. Lau JTF, Lau M, Kim JH, et al. Probable Secondary Infections in Households of SARS Patients in Hong Kong. Emerg Infect Dis 2004;10: 235-243.

25. Nao, N., Shirato, K., Katano, H., Matsuyama, S., \& Takeda, M. Detection of second case of 2019-nCoV infection in Japan (corrected version). Available online: $\quad$ https://www.who.int/docs/defaultsource/coronaviruse/method-niid-202001232.pdf?sfvrsn=fbf75320_7 (accessed on 28 January 2020)

26. Ludvigsson, J. F. Systematic review of COVID-19 in children shows milder cases and a better prognosis than adults. Acta Paediatrica 2020; 109 (6), 1088-1095.

27. Cai J, Xu J, Lin D, et al. A case series of children with 2019 novel coronavirus infection: clinical and epidemiological features. Clin Infect Dis 2020. https://doi.org/10.1093/cid/ciaa198.

28. Yan, J., Guo, J., Fan, C., Juan, J., Yu, X., Li, J., .. \& \& Lei, D. Coronavirus disease 2019 (COVID-19) in pregnant women: A report based on 116 cases. American journal of obstetrics and gynecology 2020.

29. Yang, Z., Wang, M., Zhu, Z., \& Liu, Y. Coronavirus disease 2019 (COVID-19) and pregnancy: a systematic review. The Journal of Maternal-Fetal \& Neonatal Medicine 2020; 1-4.

30. Huang, I., Pranata, R. Lymphopenia in severe coronavirus disease-2019 (COVID-19): systematic review and meta-analysis. $j$ intensive care 2020; 8: 36. https://doi.org/10.1186/s40560-020-00453-4.

31. Ai T, Yang Z, Hou H, et al. Correlation of chest CT and RT-PCR testing in coronavirus disease 2019 (COVID-19) in China: a report of 1014 cases. Radiology 2020;200642. DOI:10.1148/radiol.2020200642

32. Ye, G., Pan, Z., Pan, Y., Deng, Q., Chen, L., Li, J., \& Wang, X. Clinical characteristics of severe acute respiratory syndrome coronavirus 2 reactivation. Journal of Infection 2020.

33. Cherry, J. D., \& Krogstad, P. SARS: The First Pandemic of the 21 st Century. Pediatric research 2020; 56 (1): 1-5.

34. Shen, M., Zhou, Y., Ye, J., AL-maskri, A. A. A., Kang, Y., Zeng, S., \& Cai, S. Recent advances and 
perspectives of nucleic acid detection for coronavirus. J of Pharmaceutical Analysis 2020.

35. Qin C, Zhou L, Hu Z, et al. Dysregulation of immune response in patients with COVID-19 in Wuhan, China. Clin Infect Dis 2020;

36. Wölfel R, Corman VM, Guggemos W, et al. Virological assessment of hospitalized patients with COVID-2019. Nature 2020.

37. Rosa, S., \& Santos, W. C. Clinical trials on drug repositioning for COVID-19 treatment. Revista panamericana de salud publica = Pan American journal of public health .2020 ; 44: e40. https://doi.org/10.26633/RPSP.2020.40

38. Wu, R., Wang, L., Kuo, H. C. D., Shannar, A., Peter, R., Chou, P. J., .. \& Poiani, G. J. An Update on Current Therapeutic Drugs Treating COVID-19. Current Pharmacology Reports 2020; 1.

39. Cheng, Y., Wong, R., Soo, Y. O. Y., Wong, W. S., Lee, C. K., Ng, M. H. L., .. \& Cheng, G. (2005). Use of convalescent plasma therapy in SARS patients in Hong Kong. European Jl of Clin Microbiol and Infectious Dis 2005; 24(1): 44-46.

40. Soo, Y. O. Y., Cheng, Y., Wong, R., Hui, D. S., Lee, C. K., Tsang, K. K. S., .. \& Sung, J. J. Y. Retrospective comparison of convalescent plasma with continuing high-dose methylprednisolone treatment in SARS patients. Clin microbiol and infection 2004; 10(7): 676-678.

\section{Hosted file}

Figure.docx available at https://authorea.com/users/325047/articles/455719-sars-cov-2constitution-the-second-and-most-contagious-pandemic-of-the-21st-century 